\title{
ON MORSE-SMALE DIFFEOMORPHISMS
}

\author{
BY J. PALIS ${ }^{1}$
}

\author{
Communicated by R. S. Palais, February 14, 1968
}

Let $\operatorname{Diff}(M)$ denote the group of $C^{r}$ diffeomorphisms of a closed $C^{\infty}$ manifold $M$ endowed with the $C^{r}$ topology, $r \geqq 1$. In [6] Smale defined a subset of $\operatorname{Diff}(M)$, later called Morse-Smale diffeomorphisms, and conjectured that this subset is open and each of its elements is structurally stable.

The main purpose of this note is to announce the proof of this conjecture in the case $\operatorname{dim} M \leqq 3$, and to state some related results. These results are also valid for vector fields and then they extend results of [4] proved for the case $\operatorname{dim} M=2$.

1. Preliminaries. Following [8], for $f \in \operatorname{Diff}(M)$ we denote by $\operatorname{Per}(f)$ the set of periodic points and by $\Omega(f)$ the set of nonwandering points of $f$. A point $x \in \operatorname{Per}(f)$ of period $n$ is hyperbolic if the derivative $\left(D f^{n}\right)_{x}$ has its spectrum disjoint from the unit circle in the complex plane $C$. In this case we have the existence of stable and unstable manifolds of $x$, denoted by $W^{s}(x)$ and $W^{u}(x)$. We call $\operatorname{dim} W^{s}(x)$ the stable index of $x$.

Definition 1. $f \in \operatorname{Diff}(M)$ is called Morse-Smale if it satisfies the following conditions.

(1) $\Omega(f)$ is finite. This implies $\Omega(f)=\operatorname{Per}(f)$.

(2) All points in $\operatorname{Per}(f)$ are hyperbolic.

(3) For any pair of points $x, y \in \operatorname{Per}(f), W^{s}(x)$ and $W^{u}(y)$ are in general position.

In the sense of Smale [8], this means that $f$ satisfies Axiom A and a strong version of Axiom $\mathrm{B}, \Omega(f)$ being finite. If $f$ is Morse-Smale, the periodic orbits of $f$ can be partially ordered by the relation: $\mathcal{O}(x) \leqq \mathcal{O}(y)$ if $W^{s}(x) \cap W^{u}(y) \neq \varnothing$. The periodic orbits of $f$ with this partial order structure is called the phase-diagram of $f$, denoted by $D(f)$. By a diagram isomorphism we mean a map $\rho: D(f) \rightarrow D(g)$ which is bijective, index and order preserving.

Definition 2. $f \in \operatorname{Diff}(M)$ is structurally stable if $f$ has a neighborhood $V$ in $\operatorname{Diff}(M)$ such that for any $g \in V$ there exists a homeo. morphism $h: M \rightarrow M$ satisfying $h f(x)=g h(x)$ for all $x \in M$.

1 The results announced here are contained in the author's doctoral thesis at the University of California, written while he held a fellowship from C.N. Pq., Brazil. The author wishes to thank Professor S. Smale for his guidance. 
Definition 3. $f \in \operatorname{Diff}(M)$ is called Kupka-Smale if it satisfies conditions (2) and (3) of Definition 1.

We denote by $B(M)$ the subset of Kupka-Smale diffeomorphisms for which the following property holds:

$$
\Omega(f)=\mathrm{Cl} \operatorname{Per}(f),
$$

where $\mathrm{Cl}$ denotes topological closure. $B(M)$ is a Baire subset of $\operatorname{Diff}(M), C^{1}$ topology, see [5].

Definition 4. Let $f$ be Kupka-Smale. We say that there exists a heteroclinic phenomenon associated with $f$ if for a pair of points $x, y \in \operatorname{Per}(f), x \neq y, W^{s}(x) \cap W^{u}(y)$ has a closed nonempty component.

If $\operatorname{dim} W^{s}(x)=\operatorname{dim} W^{u}(y)$ in the above definition, then $W^{s}(x)$ $\cap W^{u}(y)$ is made up of points called heteroclinic.

\section{The results.}

THEOREM 1. The set of Morse-Smale diffeomorphisms is open in $\operatorname{Diff}(M)$. If $f$ is Morse-Smale its phase-diagram is stable (up a diagram isomorphism) under small perturbations.

We give a geometric proof of a theorem due to Hartman [2] and Grobman [1], which states that a diffeomorphism in a neighborhood of a hyperbolic fixed point is topologically conjugated to its linear part. The steps involved in this proof are useful for the following theorem.

Theorem 2. Let $\operatorname{dim} M \leqq 3$. A Morse-Smale diffeomorphism of $M$ is structurally stable.

TheOREM 3. Let $f$ be a Kupka-Smale diffeomorphism. If $f$ embeds in a topological flow then $\operatorname{Per}(f)=\mathrm{Fix}(f)$, there is no heteroclinic phenomena associated to $f$ and $f$ restricted to each invariant manifold of its periodic points is orientation preserving. For $f \in B(M)$ and $\operatorname{dim} M \leqq 2$, these conditions are also sufficient for $f$ to embed in a topological flow.

Remark. For $f \in B(M)$ the condition $\operatorname{Per}(f)=\operatorname{Fix}(f)$ implies that $f$ is Morse-Smale.

It is well known that there are diffeomorphisms near the identity map that do not embed in smooth flows. We get as immediate consequence of Theorem 3

CoRollary. In any neighborhood $U$ of the identity map in $\operatorname{Diff}(M)$, there exists an open set $V \subset U$ such that no element of $V$ embeds in a topological flow. 


\section{REFERENCES}

1. D. Grobman, Homeomorphisms of systems of differential equations, Dokl. Akad. Nauk. SSSR 128 (1959), 880-881. (Russian)

2. P. Hartman, Ordinary differential equations, Wiley, New York, 1964.

3. I. Kupka, Contribution da theorie des champs generiques, Contributions to Differential Equations 2 (1963), 457-484.

4. M. Peixoto, Structural stability on two-dimensional manifold, Topology 1 (1962), 101-120.

5. C. Pugh, An improved closing lemma and a general density theorem, Amer. J. Math. 89 (1967), 1010-1021.

6. S. Smale, Morse inequalities for a dynamical system, Bull. Amer. Math. Soc, 66 (1960), 43-49.

7. - Stable manifold for differential equations and diffeomorphisms, Ann. Scuola Norm. Sup. Pisa 17 (1963), 97-116.

8. — Differentiable dynamical systems, Bull. Amer. Math. Soc. 73 (1967), 747-817.

University of California, Berkeley 